\title{
Tunable Attractions Directing Nonequilibrium States in Dispersions of Hard Rods
}

\author{
M. P. B. van Bruggen ${ }^{\dagger}$ and H. N. W. Lekkerkerker* \\ Van't Hoff Iaboratory for Physical and Colloid Chemistry, Debye Institute, Utrecht University,
} Padualaan 8, $3584 \mathrm{CH}$ Utrecht, The Netherlands

Received February 2, 2000

\begin{abstract}
The effect of attractive interactions on the phase behavior of colloidal hard rods has been studied by the addition of nonadsorbing polymer. In these rod-polymer mixtures, four pathways of phase separation can be distinguished: nucleation and growth, spinodal decomposition, aggregation, and gelation. The first three mechanisms give rise to macroscopic phase separation, whereas for the latter mechanism this is not necessarily the case. The observed phenomena are explained on the basis of an interplay between the position of both the spinodal line and the glass line, the overall composition of the sample, and the range of the potential.
\end{abstract}

\section{Introduction}

Fifty years ago Onsager ${ }^{1}$ showed that the isotropicnematic phase transition in dispersions of repulsive rodlike particles can be understood on the basis of a decrease in excluded volume compensating a loss of orientational entropy. Today there are a considerable number of experiments that can be described semiquantitatively with the Onsager theory and its extensions. ${ }^{2}$

Recently we studied the morphology and kinetics of the isotropic-nematic phase transition in a dispersion of sterically stabilized boehmite rods in cyclohexane, which to a good approximation can be considered as hard rods. ${ }^{3}$ Increasing the concentration of the rods through the biphasic isotropic-nematic region, we observed a crossover from nucleation and growth to spinodal decomposition as predicted by Doi and coworkers. ${ }^{4}$

Attractive interactions in dispersions of rodlike particles and macromolecules easily bring about aggregation and gelation. ${ }^{5-8}$ These metastable states often obscure large parts of the equilibrium phase diagram. In this paper we present experiments on model dispersions of hard rods in which the attractive interactions are switched on by adding nonadsorbing polymer. Rods that come closer than the size of the added polymers experience an attraction due to an uncompensated osmotic pressure of the polymers. ${ }^{9,10}$ The effective attraction is called depletion attraction and is wellknown in colloid science. ${ }^{11}$ The range of the depletion attraction is directly related to the size (i.e., molecular mass) of the polymers, and the strength is proportional to the polymer concentration. In this study we try to show that by fine-tuning the attractive interactions in this way nonequilibrium states are engendered, the manifestation of which can be closely related to the theoretical equilibrium phase diagram of the rodpolymer mixture.

The outline of this paper is as follows. In section 2 experimental details are given, followed by the results in section 3. Section 4 gives the discussion of the results, and it is concluded with a brief summary in section 5 .

${ }^{\dagger}$ Current address: Philips Research, Prof. Holstlaan 4, 5656AA Eindhoven, The Netherlands.

* To whom correspondence should be addressed.
Table 1. Length and Diameter of the Colloidal Rods As Determined with TEM

\begin{tabular}{ccc}
\hline$L(\mathrm{~nm})$ & $\mathrm{D}(\mathrm{nm})$ & $\mathrm{L} / \mathrm{D}$ \\
\hline 250 & 17 & 15
\end{tabular}

\section{Experimental Section}

2.1. Polyisobutylene Grafted Boehmite Rods. Aqueous dispersions of charge stabilized boehmite rods were transferred to 1-propanol and grafted with modified polyisobutylene (PIB). The synthesis of boehmite rods and the grafting procedure are described in refs 12 and 13. The PIB (provided by Shell) consists of a polyalkylamine anchor group that is chemically connected to two polyisobutylene tails with a molecular weight of $1000 \mathrm{~g} / \mathrm{mol}$ each. The PIB grafted rods were dispersed in cyclohexane (Merck, p.a.). The dimensions of the rods were determined with transmission electron microscopy (TEM), and the results are given in Table 1.

The polydispersity of the sterically stabilized rods is $25 \%$ in both length and diameter. The diameter also includes the length of the polymer tail length $\mathrm{h}$, which is here about $4 \mathrm{~nm}$. The volume fraction of rods is corrected for the fact that the PIB chains enclose solvent. When the rods are much longer than the length $\mathrm{h}$ of the PIB chains, the correct volume fraction $\phi$ is given by

$$
\phi=\frac{\mathrm{C}}{\rho_{\mathrm{b}}(1+\mathrm{x})}\left(\frac{\mathrm{D}+\mathrm{h}}{\mathrm{D}}\right)^{2}
$$

where $c$ is the mass concentration of the PIB grafted boehmite rods in the dispersion, $D$ is the diameter of the rods, $\rho_{b}$ is the density of the bare boehmite rods $\left(3.0 \mathrm{~g} / \mathrm{mL}^{13}\right)$, and $x$ the mass ratio of PIB to boehmite (typically 0.25 ). The mass concentration was determined by drying dispersions at $60{ }^{\circ} \mathrm{C}$ in a nitrogen flow.

2.2. Rod-Polymer Mixtures. The influence of attractive interactions on the phase separation process was studied by adding stock solutions of poly(dimethylsiloxane) (PDMS) in cyd ohexane to samples of the rod dispersion. The polymer used here had a molar weight $M_{w}$ of $91.7 \mathrm{~K}$ ( $A B C R$ made), and the polydispersity is expressed by $M_{w} / M_{n}$, where $M_{w}$ is the weightaverage and $\mathrm{M}_{\mathrm{n}}$ is the number-average mol ecular weight. This ratio here is 1.30. The radius of gyration $R_{g}$ in cycl ohexane is $13 \mathrm{~nm}$. The effective polymer volume fraction (i.e., volume fraction of polymer coils) $\phi_{\text {eff }}$ is calculated by

$$
\phi_{\text {eff }}=\frac{\mathrm{CN}_{\mathrm{av}}}{\mathrm{M}_{\mathrm{w}}} \frac{4}{3} \pi \mathrm{R}_{\mathrm{g}}^{3}
$$

where $\mathrm{c}$ is the polymer concentration and $\mathrm{N}_{\mathrm{av}}$ is Avogadro's number. 


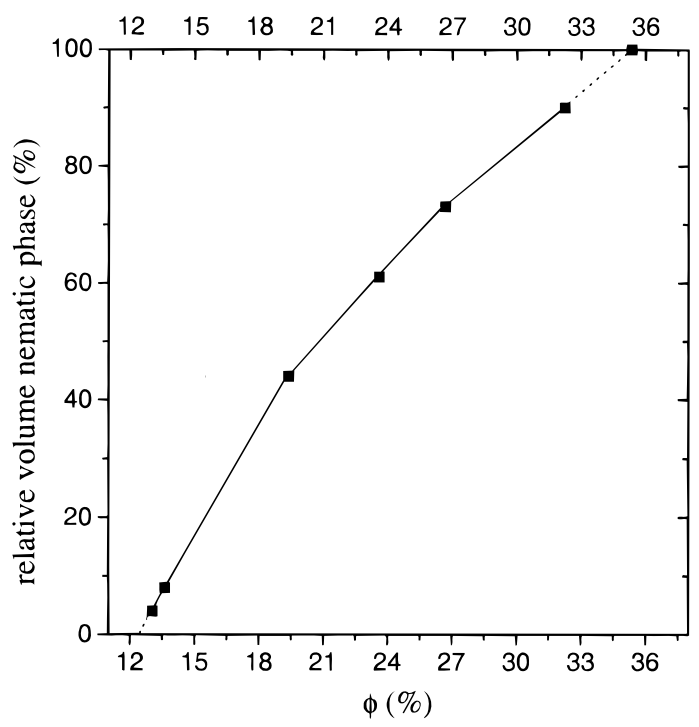

Figure 1. Experimental phase diagram of sterically stabilized boehmite rods dispersed in cycl ohexane. The phase boundaries are obtained by extrapolating the solid line to a relative nematic volume of respectively $0 \%$ and $100 \%$.

To three monophasic, isotropic samples different amounts of pure PDMS were added. Sample A contained $10.3 \%$ rods and $3.4 \mathrm{~g} / \mathrm{L}$ PDMS, corresponding to an effective PDMS volume fraction of $29 \%$. System B contained $10.1 \%$ rods and $6.0 \mathrm{~g} / \mathrm{L}$ PDMS, which is equivalent to an effective coil volume fraction of $51 \%$. Sample C had a rod volume fraction of $10 \%$ and contained $24 \mathrm{~g} / \mathrm{L}$ PDMS. Finally, sample D had a rod concentration of $22 \%$, which is in the biphasic region of the pure rod system, and had a PDMS concentration of $0.75 \mathrm{~g} / \mathrm{L}$.

2.3. Polarization Light Microscopy. The phase separation process was followed with a polarization mi croscope (Zeiss axioplan microscope). Tubes containing different samples of the dispersion were homogenized by vortexing and subsequently transferred into glass cuvettes (Vitrodynamics) with an optical path of $100 \mu \mathrm{m}$. After sealing the cuvettes, the samples were examined under the polarization microscope.

\section{Results}

3.1. Hard Rod System. The phase diagram of the hard rod system without adding polymer has been determined earlier ${ }^{8}$ and is given in Figure 1 . Above a rod volume fraction of about $12 \%$ the dispersion starts separating into an isotropic phase and a nematic phase, Above $35 \%$ the dispersion is fully nematic. The fact that the determined phase diagram of this system extends over a broader concentration region than predicted by computer simulations ${ }^{14}$ is attributed to the polydispersity of the rods. ${ }^{15}$

3.2. Rod-Polymer Mixtures. We now summarize the observations by the eye as well as the findings as reveal ed by the polarization microscope.

Sample A. The turbidity of this sample slowly increased in time. One minute after homogenization, small $(1-10 \mu \mathrm{m})$ birefringent droplets appeared under the polarization microscope. When the sample was illuminated by a laser beam, a clear light scattering ring at a few degrees was visible on the wall. The smooth droplets grew within minutes to sizes of tens of micrometers and sedimented to the bottom. Within a few hours a strongly birefringent phase started growing from the bottom. After 1 day macroscopic phase separation was complete. The bottom phase consisted of a liquidlike birefringent phase; the upper phase was only streaming birefringent and is therefore considered to be an isotropic dispersion.
Sample B. After homogenization this sample became immediately very turbid. One minute after homogenization, small birefringent droplets appeared again under the polarization mi croscope. So initially A and B looked very similar. However, after some longer time when the initial droplets had grown larger, they appeared to have very irregular surfaces. These irregular domains associated by sticking to each other (instead of coalescing), finally forming an irregular stacking of aggregates which grew from the bottom of the cuvette. Probably due to the high turbidity, only a diffuse light scattering ring could be observed. After 1 day macroscopic phase separation was complete. The bottom phase did not flow upon tilting the test tube; it was very turbid and very weakly birefringent. The upper phase was an isotropic dispersion.

Sample C. The turbidity of this sample was immediately extremely high, and the whole sample appeared to be weakly bir efringent when studied between crossed polarizers. Under noncrossed polarizers, a regular transmission variation became visible under the microscope. This indicates that the density of the sample was inhomogeneous but varied periodically in space. This morphology did not seem to change in time anymore. However, after 1 day, a nonbirefringent and opaque phase was shrinking from the miniscus, leaving a clear supernatant.

Sample D. As for sample A the turbidity of the homogenized dispersion slowly increased in time. Its microstructure as seen under the microscope roughened and coarsened. Instead of forming discrete droplets as in $A$, a bicontinuous structure was being formed. Like sample A, this samplealso devel oped a ring-shaped light scattering pattern. Within a few hours a birefringent phase started growing from the bottom of the cuvette. After 1 day macroscopic phase separation was complete. The bottom phase was liquidlike and highly birefringent whereas the upper phase was isotropic.

Polarization micrographs taken during phase separation of the four samples are given in Figure 2.

Referring to our earlier study on pure hard rod dispersions, ${ }^{13}$ the morphological development as observed in sample A is identified as a nucleation and growth process. Phase separation in sample B initially looks as nucleation and growth, but here the nuclei are aggregated. Therefore, the phase separation process of sample $B$ is denoted as aggregation. The bicontinuous structure in sample D is a typical example of spinodal decomposition. The bicontinuous morphology of $\mathrm{C}$ resembles $D$, but here the structural development is arrested after a short time. The system is clearly gelled. Compared to the isotropic phase of sample B, the transmission of the isotropic phases in samples $A$ and $D$ is smallest. This indicates that the concentrations of rods in these coexisting isotropic phases are the highest. The upper phase of sample $C$ is transparent and does not seem to contain any rods. The nematic phase volume in $D$ is almost equal to the nematic fraction formed in a sample with the same rod concentration, without PDMS. This indicates that the biphasic region has not yet widened much at this polymer concentration (see discussion).

On the basis of the above given observations and identifications, the binodal of the nematic phase and the tie lines are sketched in Figure 3. In this figure also the binodal of the isotropic phase as determined by Buitenhuis $^{8}$ is given. The composition of the samples 
a

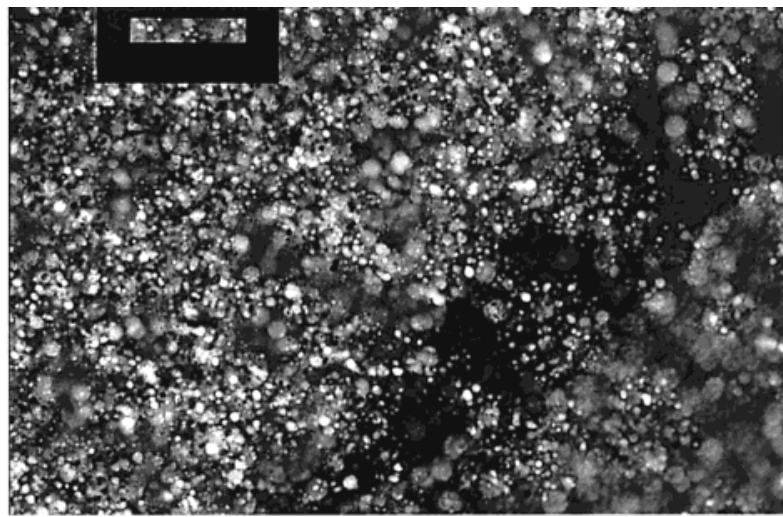

b

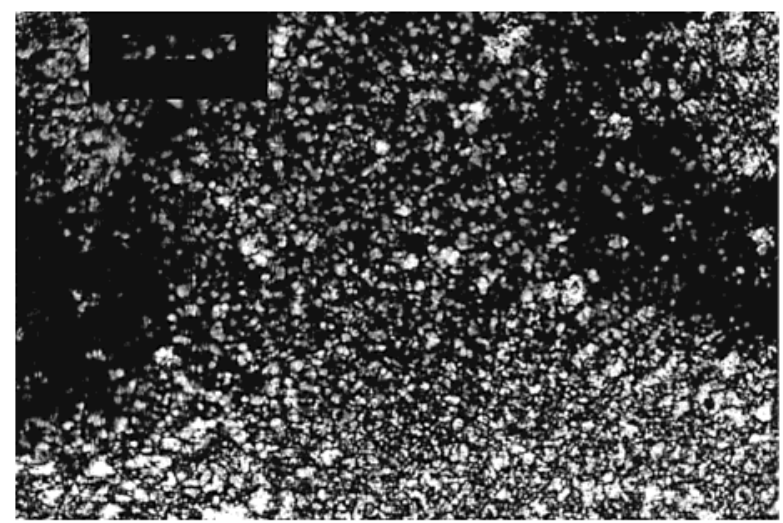

c

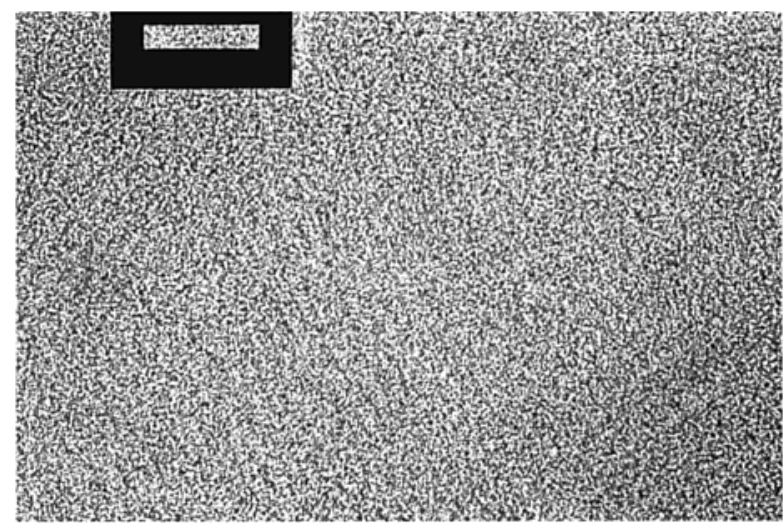

d

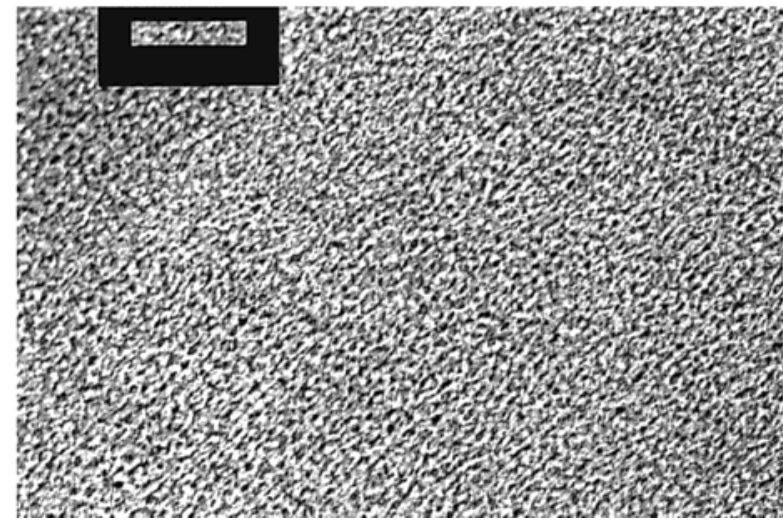

Figure 2. Micrographs between crossed $(a, b, d)$ and noncrossed (c) polarizers of rod-polymer mixtures with compositions given in Table 2: (a) nucleation and growth in sample $A$ photographed after $60 \mathrm{~min}$, (b) aggregation of separate droplets in sample B after $140 \mathrm{~min}$, (c) pinned spinodal decomposition in sample $\mathrm{C}$ after $60 \mathrm{~min}$, and (d) spinodal decomposition in sample D after $9 \mathrm{~min}$. The bar represents $200 \mu \mathrm{m}$; in (c) this is $100 \mu \mathrm{m}$

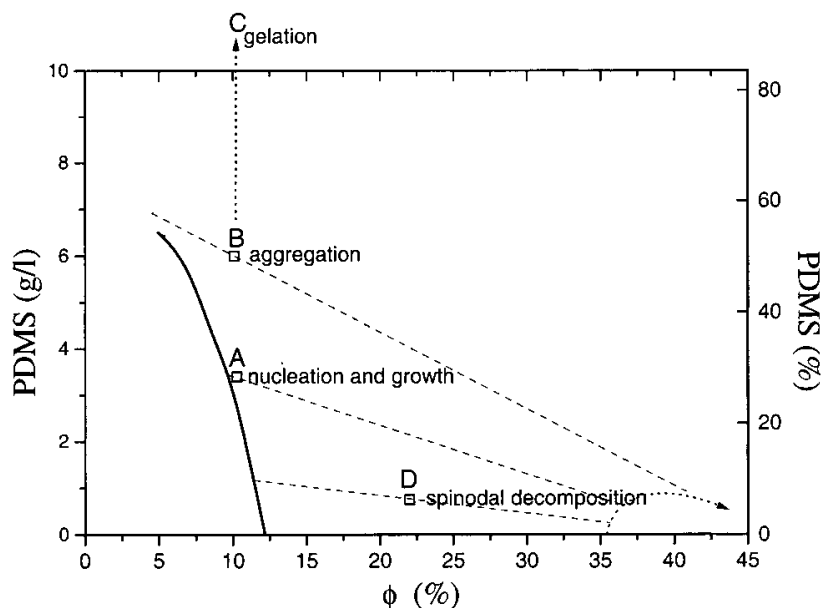

Figure 3. Experimentally determined binodal (solid line) of the isotropic phase of sterically stabilized boehmite rods mixed with PDMS. ${ }^{8}$ The dotted line is a sketch of the binodal of the nematic phase, and the dashed line represents the supposed tie lines. The points $A-D$ give the location of the experimentally prepared samples. System C falls of the y-scale of this figure; this sample contained $24 \mathrm{~g} / \mathrm{L}$ PDMS.

Table 2. Data on the Rod-Polymer Mixtures

\begin{tabular}{cccl}
\hline & $\begin{array}{c}\text { rod volume } \\
\text { fraction (\%) }\end{array}$ & $\begin{array}{c}\text { PDMS concn } \\
(\mathrm{g} / \mathrm{L})\end{array}$ & $\begin{array}{c}\text { phase separation } \\
\text { morphology }\end{array}$ \\
\hline A & 10.3 & 3.4 & nucleation \\
B & 10.1 & 6.0 & aggregation \\
C & 9.3 & 24.0 & gelation \\
D & 22.0 & 0.75 & spinodal decomposition
\end{tabular}

and the observed morphological development are summarized in Table 2. Buitenhuis also noted that bire fringent bottom phases were only formed at rod volume fractions close to the isotropic binodal and not too high polymer concentration. At lower volume fractions the bottom phase appeared to be nonbirefringent and gellike.

\section{Discussion}

Poon, Pirie, and Pusey ${ }^{16}$ related the transition from crystallization to gelation in mixtures of colloidal spheres and polymers to the crossing of a "hidden" metastable isotropic-isotropic binodal. In a preliminary study on rod-polymer mixtures, similar arguments were put forward. ${ }^{17}$ I nspired by the work of Frank and Keller, ${ }^{18}$ who explained gelation in relation to fluid-fluid separation, as well as by recent work on the kinetics of the isotropic-nematic phase separation, ${ }^{3}$ we here present a different point of view in this matter.

Consider a mixture of colloidal rods and nonadsorbing polymers in osmotic equilibrium with a reservoir containing only the nonadsorbing polymers. Theoretical phase diagrams for such systems have been calculated by Lekkerkerker and Stroobants. ${ }^{19}$ A sketch of such a phase diagram containing the features as displayed by the current experimental system is given Figure 4. The polymer concentration in the reservoir is given on the $y$-axis, so the tie lines connecting the coexisting phase are horizontal. The reservoir concentration plays a role analogous to the inverse of the temperature in thermotropic liquid crystal systems. In this phase diagram the overall compositions of the experimental systems A, B, $C$, and D are indicated by the solid dots and the coexisting phases by the open circles. The spinodal line delineating nucleation and growth from spinodal de composition is also drawn schematically. Theoretical 


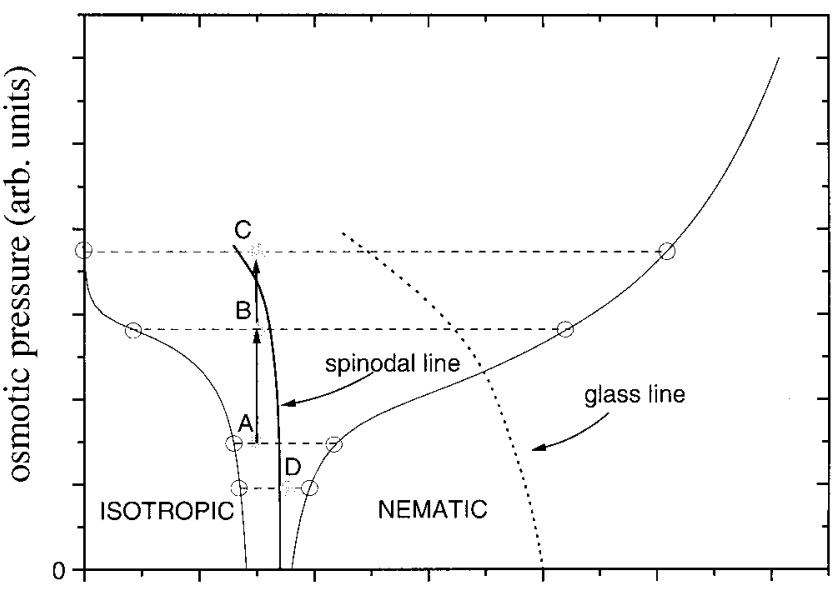

$\phi$

Figure 4. Phase diagram of an arbitrary rod polymer mixture. On the $y$-axis the osmotic pressure of the polymer reservoir is given, and the $x$-axis gives the rod volume fraction. Samples $A-D$ (dots) are plotted in this phase diagram, together with the glass line and the spinodal line. The open circles represent the coexisting phases which are connected to each other by the dashed tie lines.

evidence for a spinodal point in a pure hard rod system was already given by Onsager ${ }^{1}$ and elaborated by Doi and co-workers, and experimental work confirms these predications. ${ }^{3}$ At concentrations right from the dotted line in Figure 4, the motion of the rods has ceased due to entanging of the rods. This line will be referred to as the glass line. Like the temperature dependence of the glass line as being put forward by Keller, ${ }^{18}$ it is here assumed that the glass line bends to lower rod concentrations when the osmotic pressure of the nonadsorbing polymers increases.

First consider systems $A$ and $D$. The overall composition of system $A$ is left from the spinodal and phase separation occurs by nucl eation of droplets, whereas in $\mathrm{D}$ it occurs by spi nodal decomposition. The composition of both the coexisting phases of $A$ and $D$ are at the left of the glass line, and the nematic phases will reach their equilibrium composition. The overall concentration of system $B$ is still left from the spinodal so the phase separation process proceeds by nucleation of droplets. However, the concentration of the coexisting nematic phases in B is above the glass line, and consequently the densifying nematic becomes arrested before its equilibrium state is reached. Because the rods are not able to rearrange inside the droplets, the interface of the droplets cannot adjust in such a way that the surface free energy is minimized. As a consequence, the frozen droplets stick rather than coalesce, finally yielding irregular aggregates as depicted in Figure $2 \mathrm{~b}$. In C spinodal decomposition governs the phase separation process, and a bicontinuous density profile develops. However, when the density in the rod-rich phase passes the glass line, the dynamics of rods is frozen, and the spinodal structure makes that the entire system solidifies. We remark that although the density has segregated into high and low regions, the orientational ordering of the rods is still isotropic. Both the density difference between the two coexisting phases and the rigidity of the frozen-in spinodal structure will determine the lifetime of the gel in $\mathrm{C}$. The scenario that is encountered in other phase separating systems will depend on the location of both the spinodal line and the glass line.
The range of the potential plays an important role here since it determines whether the two-phase region broadens to the high concentration side or the low concentration side. The calculations of Lekkerkerker and Stroobants ${ }^{19}$ show that when the radius of gyration of the nonadsorbing polymers is small compared to the radius of the rods, the formation of concentrated ne matics is enhanced. The rationalization of this is that rods with narrow potentials can only reduce the free energy when they are close enough together. For broader potentials, as is the case in the present study, the formation of less dense phases is enhanced. Consequently, gelation during phase separation is likely to occur when the range of the potential is small. In this case the equilibrium concentration of the nematic will readily exceed the glass line. To test this hypothesis, additional experiments are required in which the range is systematically varied by using high- and low-molecular weight polymers.

\section{Summary}

In this paper the effect of attractive interactions on the isotropic-nematic phase separation was studied. In phase separating dispersions of hard rods where the attractions are tuned by the concentration of nonadsorbing polymer, depletion attraction can cause either nucleation and growth, spinodal decomposition aggregation, or gelation. This can be rationalized in terms of a rod-polymer phase diagram together with the spinodal line and the glass line. This model indicates under what conditions dispersions of attractive rods will either gel or aggregate and in addition why short-range potentials disfavor the formation of equilibrium states.

Acknowledgment. This work was supported by The Netherlands Foundation for Chemical Research (SON) with financial aid from The Netherlands Organization for Scientific Research (NWO).

\section{References and Notes}

(1) Onsager, L. Ann. N.Y. Acad Sci. 1949, 51, 627.

(2) Vroege, G. J .; Lekkerkerker, H. N. W. Rep. Prog. Phys. 1992, $55,1241$.

(3) van Bruggen, M. P. B.; Dhont, J . K. G.; Lekkerkerker, H. N. W. Macromol ecules 1999, 32, 2256.

(4) Shimada, T.; Doi, M.; Okano, K. J . Chem. Phys. 1988, 88, 7181.

(5) Heller, W. J . Phys. Chem. 1941, 45, 1203

(6) Bernal, J. D. Trans. Faraday Soc. 1946, 42B, 1.

(7) Russo, P. S.; Miller, W. G. Macromolecules 1983, 16, 1690.

(8) Buitenhuis, J .; Donselaar, L. N.; Buining, P. A.; Stroobants, A.; Lekkerkerker, H. N. W. J . Colloid Interface Sci. 1995 $175,46$.

(9) Asakura, S.; Oosawa, F. J . Chem. Phys. 1954, 22, 1255

(10) Asakura, S.; Oosawa, F. J . Polym. Sci. 1958, 33, 183.

(11) Vrij, A. Pure Appl. Chem. 1976, 48, 471.

(12) Buining, P. A.; Pathmamanoharan, C.; J ansen, J. B. H.; Lekkerkerker, H. N. W. J . Am. Ceram. Soc. 1991, 74, 1303.

(13) Buining, P. A.; Veldhuizen, Y. S. J .; Pathmamanoharan, C.; Lekkerkerker, H. N. W. Colloids Surf. 1992, 64, 47.

(14) Bolhuis, P. G.; Frenkel, D. J . Chem. Phys. 1997, 107, 1551.

(15) Vroege, G. J .; Lekkerkerker, H. N. W. J . Phys. Chem. 1993, 97, 3601.

(16) Poon, W. C. K.; Pirie, A. D.; Pusey, P. N. Faraday Discuss. 1996, 101, 65.

(17) van Bruggen, M. P. B.; van der Kooij, F. M.; Lekkerkerker, H. N. W. J. Phys.: Condens. Matter 1996, 8, 9451.

(18) Frank, F. C.; Keller, A. Polym. Commun. 1988, 29, 186.

(19) Lekkerkerker, H. N. W.; Stroobants, A. II Nuovo Cimento 1994, 16D, 949.

MA0001943 